\title{
Low-Grade Fibromyxoid Sarcoma of the Renal Pelvis: First Report
}

\author{
Aristeidis Alevizopoulos Vasilis Mygdalis Stayros Tyritzis \\ K. Stravodimos Constantinos A. Constantinides
}

1st Academic Urology Department of Athens, Athens University Medical School - LAIKO Hospital, Athens, Greece

\section{Key Words}

Kidney sarcoma $\cdot$ Low-grade fibromyxoid sarcoma of renal pelvis $\cdot$ Renal pelvis sarcoma $\cdot$ Soft tissue sarcoma

\begin{abstract}
Sarcomas of the genitourinary tract are quite rare, accounting for $2.1 \%$ of all soft tissue sarcomas and have a poor prognosis. Kidney sarcomas are quite rare, representing 1-3\% of malignant renal cases. Low-grade fibromyxoid sarcoma (LGFS) of the kidney is an exceedingly uncommon, indolent but metastasizing soft tissue sarcoma with deceptively benignappearing histological features. The estimated 5 -year overall survival seems to be over $90 \%$, but very late local relapses and distant metastasis may occur, which underlines the need for a long-term follow-up. We present a case of a 48-year-old male patient with a LGFS located on the renal pelvis. This is probably the first report of LGFS arising from the renal pelvis.
\end{abstract}

\section{Introduction}

Soft tissue sarcomas (STS) are a heterogeneous group of rare tumors that arise predominantly from the embryonic mesoderm and account for about $1 \%$ of all malignant tumors, with the genitourinary (GU) tract accounting for $2.1 \%$ of STS [1-3]. Sarcomas of the kidney are quite rare [4]. They represent between 1 and $3 \%$ of all renal cancer cases and have a poor prognosis $[4,5]$. Early diagnosis and surgical resection offer patients the best chance of survival [4].

Low-grade fibromyxoid sarcoma (LGFS) is a rare indolent but metastasizing STS, with deceptively benign-appearing histological features. The most common tumor locations are the shoulder area, thigh and inguinal area and the primary tumor presents 
as a painless growing mass. LGFS of the kidney is exceedingly uncommon [8]. To our knowledge this is the first report of LGFS arising from the renal pelvis.

The treatment of choice for LGFS consists of surgery. Radiation and chemotherapy are used in addition to surgery in the treatment of recurrent and metastatic tumor depending on the situation.

\section{Case Report}

A 48-year-old male patient consulted the urology department of our hospital, reporting a recent episode of left renal colic pain. He denied having any symptoms from the lower urinary tract. Past medical history is significant for surgical removal of parathyroid glands due to hyperparathyroidism, accompanied by recurrent kidney stone formation, which was treated by sessions of extracorporeal lithotripsy (ESWL). The patient's physical examination was normal. Laboratory tests (blood count, blood biochemistry) were within normal limits, while the urinanalysis has shown the presence of 2030 RBC per high-power field, which was considered in concordance with the renal colic pain. Urine cytology showed a nonspecific cellular atypia, while chest X-rays were normal. An ultrasound exam was performed as a first diagnostic imaging approach, which found the presence of a $4.5-\mathrm{cm}$ renal pelvic mass on the left kidney. A computed tomography (CT) exam was recommended to the patient and the result consisted of renal stones on both kidneys, and a soft tissue mass, mostly located inside the left renal pelvis, of $4.3 \times 4 \times 4.6 \mathrm{~cm}$ (fig. 1 ). The patient was subsequently subjected to ureteroscopy, which confirmed the presence of a large exophytic mass, which occupied a big part of the renal pelvis, with apparent characteristics of malignant tumor. A tumor biopsy specimen was taken for histologic examination, and the pathological report confirmed the presence of a renal pelvis tissue with a dysplastic urothelium. The subepithelial tissue immunohistologic examination [Panker(-), S100(-), CD34(+) and SMA(+)] was indicative of hyperplasia of smooth muscle, with no apparent evidence of malignancy. Even though the pathological result was benign, the apparent malignant macroscopic characteristics of the tumor, in combination with a detailed consultation with the patient, pointed to the decision of an open left radical nephroureterectomy, which was performed 5 days after the ureteroscopy. The intervention took place uneventfully, the postoperative period was uncomplicated and the patient was discharged 4 days after the operation. The surgical pathology report described the presence of a myxomatoid tumor of $4.7 \mathrm{~cm}$ in diameter in the renal pelvis. Histologically, the tumor had a compact structure showing an admixture of hypocellular myxoid and hypercellular collagenized zones, consisting mostly of delicate spindle-shaped cells ( $\underline{\text { fig. } 2}$, fig. 3 ). Mitoses were rare. Arcades of blood vessels were also seen, while giant areas of hyalinizing collagen were seen. The tumor was in contact with the renal pelvis urothelial wall through a thin fibrous capsule, without infiltrating the urothelium. The immunohistochemical results [vimentin(+), SMA(-), c-kit(focally weak positivity), desmin(-), S-100(-), EMA(-), bcl2(-), CD31(-), CD99(-), CD34(+) and Ki-67 = 1\%], demonstrated the presence of a malignant mesenchymal tumor of the renal pelvis, strongly suggesting the diagnosis of LGFS [10]. The specimen's surgical margins were free of tumor infiltration.

The patient was referred to an oncologist for a second opinion and a close follow-up was decided, without any adjuvant therapy. The patient is until now (7 months of follow-up) free of local recurrence, or metastatic disease.

\section{Discussion}

Renal sarcomas are quite rare tumors with less than 150 cases reported in the literature [8]. Silverman et al. [9] reported the first LGFS of the renal capsule (capsuloma). We believe our case is the first to report a LGFS of the renal pelvis.

LGFS is an uncommon soft tissue malignancy. It is also known as 'Evans tumor', because the first 2 cases were reported by Evans in 1987, followed by a series of 33 
cases in a recent report [6, 7]. In these series LGFS occurred mostly in young adults with almost even sex distribution. Tumor size ranged from 1.5 to $16 \mathrm{~cm}$, while the operated tumors were estimated to be 1 month to 25 years old. Local recurrences were noted at intervals varying from 0 to 15 years and metastases developed after intervals ranging from 0 to 45 years and were mostly located in the lungs, pleura and chest wall [6]. Although the estimated 5-year overall survival exceeds $90 \%$, these findings underline the need for a long-term follow-up of the patients, and a more detailed evaluation of the tumor's behavior [7]. The primary treatment of choice is surgical removal of the tumor. Although the tumor prognosis has been improved due to the early radical surgery, the disease-specific mortality is obscure, because of the tumor capability of distant metastasis, even 50 years after surgery [7]. Radiotherapy and chemotherapy can be used additionally when recurrences or metastases occur. Negative surgical margins and tumor size seem to be the most important accepted prognostic factors for better survival and local control of the tumor [7].

\section{Conclusion}

LGFS of the kidney is a rare soft tissue neoplasm entity that can have a deceptively indolent histologic appearance but clearly malignant course. Surgery is the treatment of choice and long-term follow-up is implicit due to late local and metastatic occurrences. Radiotherapy and chemotherapy should be reserved for local recurrence and/or local metastasis treatment. To our knowledge this is the first case report of a LGFS of the renal pelvis.

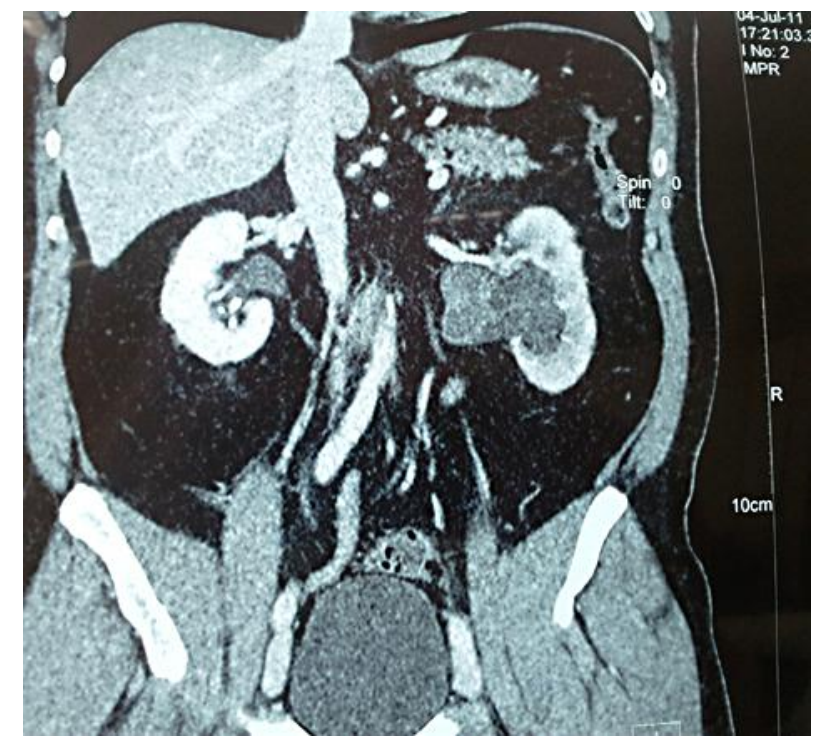

Fig. 1. CT showing left renal pelvis mass. 


\section{Case Reports in Nephrology and

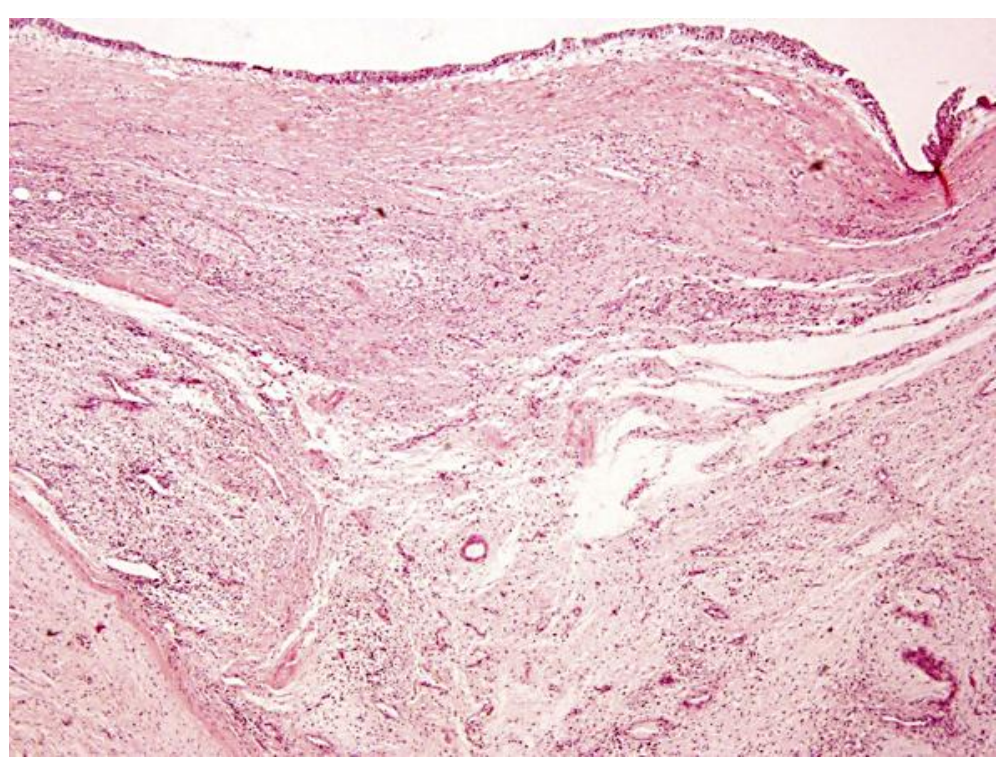

Fig. 2. Low-grade fibromyxoid sarcoma covered by pelvic urothelium. HE stain $(\times 40$ original magnification).

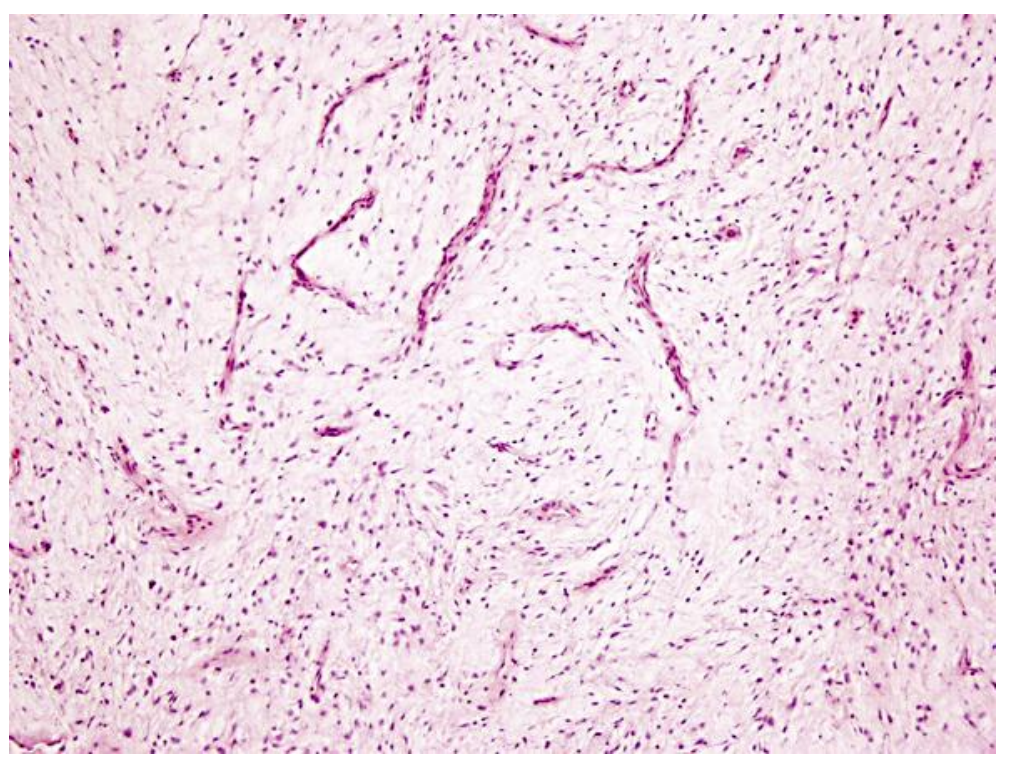

Fig. 3. Low-grade fibromyxoid sarcoma. Myxoid area showing arcades of blood vessels. HE stain $(\times 200$ original magnification). 


\section{References}

1 Russo P, Brady MS, et al: Adult urological sarcoma. J Urol 1992;147:1032.

$>2$ Hayes AJ, Thomas JM: Soft tissue tumours. Postgrad Med J 1997;73:705-709.

-3 Stojadinovic A, Leung DH, Allen P, Lewis JJ, Jaques DP, Brennan M: Primary adult soft tissue sarcoma: time-dependent influence of prognostic variables. J Clin Oncol 2002;20:4344.

4 Dotan ZA, Tal R, Golijanin D, et al: Adult genitourinary sarcoma: the 25-year Memorial Sloan-Kettering experience. J Urol 2006;176:2033-2038.

$\checkmark 5$ Cho SY, Moon KC, Cheong MS, Kwak C, Kim HH, Ku JH: Localized resectable genitourinary sarcoma in adult Korean patients: experiences at a single center. Yonsei Med J 2011;52:761-767.

$\checkmark 6$ Evans HL: Low-grade fibromyxoid sarcoma: a report of two metastasizing neoplasms having a deceptively benign appearance. Am J Clin Pathol 1987;88:615-619.

7 Evans HL: Low-grade fibromyxoid sarcoma: a clinicopathologic study of 33 cases with long-term followup. Am J Surg Pathol 2011;35:1450-1462.

8 Murphy WM, Beckwith JB, Farrow GM: Atlas of Tumor Pathology. Tumors of the Kidney, Bladder, and Related Urinary Structures. Washington, DC, Armed Forces Institute of Pathology, 1993, pp 153-163.

>9 Silverman JF, Nathan G, Olson PR, Prichard J, Cohen JK: Fine-needle aspiration cytology of low-grade fibromyxoid sarcoma of the renal capsule (capsuloma). Diagn Cytopathol 2000;23:279-283.

10 Fletcher C, Unni K, Mertens F: Pathology and Genetics of Tumours of Soft Tissue and Bone. Lyon, IARC, 2002, pp 104-105. 